\title{
GOBIERNO CORPORATIVO Y DESEMPEÑO FINANCIERO DE LAS EMPRESAS CHILENAS
}

\section{CORPORATE GOVERNANCE AND FINANCIAL PERFORMANCE OF CHILEAN COMPANIES}

\author{
Hugo Moraga Flores ${ }^{1}$ y Eva Ropero Moriones $^{2}$
}

\section{RESUMEN}

El objetivo de la presente investigación es determinar y analizar el grado de adopción y homogeneidad de las prácticas de gobierno corporativo (en adelante GC) implementadas por las empresas chilenas inscritas en la Superintendencia de Valores y Seguros (SVS), a través del análisis de las respuestas al cuestionario exigido por la Norma de Carácter General N³85, además de establecer, si esta adopción afecta a variables de desempeño financiero de las empresas en revisión. Los datos analizados corresponden a las respuestas entregadas por las propias empresas a la SVS, para el año 2015 que se divulgaron a través de la página web de la SVS el año 2016.

Para el logro del objetivo propuesto, hemos elaborado un indicador de adopción de prácticas de GC que hemos tomado como variable para establecer si el sector y subsector económico de pertenencia, tiene alguna influencia en el grado de adopción de prácticas de GC obtenido por las empresas que conforman la base de datos en análisis. Para dicho efecto se realizó un estudio descriptivo y se aplicaron herramientas estadísticas como el coeficiente de variación (CV), con lo cual se determinó que dichos grupos eran heterogéneos en la adopción, posteriormente se realizó un análisis de normalidad al grupo de datos lo cual estableció que se debían aplicar pruebas no paramétricas, utilizando la Rho de Spearman.

A través de nuestra investigación, establecimos que no existe una relación significativa entre el grado de adopción y la valorización de la empresa medida a través de la Q de Tobin, la variable de rentabilidad y retorno sobre el patrimonio, pero logramos establecer relación entre la pertenencia a los indicadores, Dow Jones Sustainability Index Chile e Índice de Precios Selectivo de Acciones (IPSA).

Palabras clave: Gobiernos Corporativos, Desempeño financiero, RSE, SVS.

Recepción: 22/01/2018. Aprobación: 26/06/2018.

\begin{abstract}
The purpose of this research is to determine and analyze the degree of adoption and homogeneity of the corporate governance practices (hereinafter GC) implemented by the Chilean companies registered with the SVS, through the analysis of the Answers to the questionnaire required by General Standard No. 385, in addition to establishing, if this adoption affects variables of financial performance of the companies under review. The data analyzed correspond to the responses provided by the companies themselves to the SVS, for the year 2015, which were disseminated through the SVS website in 2016.
\end{abstract}

1 Escuela de Contador Auditor, Facultad de Economía y Negocios, Universidad Andrés Bello, Concepción, Chile. E-mail: hmoraga@unab.cl 
In order to achieve the proposed objective, we have developed an indicator of adoption of GC practices that we have taken as a variable to establish whether the sector and economic subsector of membership has any influence on the degree of adoption of GC practices obtained by companies that make up the database under analysis. For this effect, a descriptive study was carried out and statistical tools such as the coefficient of variation $(\mathrm{CV})$ were applied, which determined that these groups were heterogeneous in adoption, after which a normality analysis was performed to the data group, which established That nonparametric tests should be applied using Spearman's Rho.

Through our research, we established that there is no significant relationship between the degree of adoption and the valuation of the company measured through the $Q$ of Tobin, the variable of return and return on equity, but we managed to establish a relationship between membership to the indicators, Dow Jones Sustainability Index Chile and Selective Stock Price Index (IPSA).

Keywords: Corporate Governance, CSR, financial performance, SVS.

\section{INTRODUCCIÓN}

El aumento en los requerimientos de información, ya sean voluntarios o exigidos, producto de las crisis financiera a los que se han visto enfrentado los países latinoamericanos, como los casos de corrupción y / o colusión en las cuales han participado importantes empresas del país, han llevado a que los consumidores e inversionistas o stakeholders en general, requieran mayor información para la toma de decisión, ya sea de consumo o de inversión (Rivero, 2005).

El tema de Responsabilidad Social Empresarial (en adelante RSE), es un tema que ya está asumido por la mayoría de los países y por las diferentes organizaciones, pero este tema tiene varias aristas, las cuales en un principio deben ser tratadas en forma independiente, pero que deben promover un mismo objetivo, la sostenibilidad de la empresa. Sumado a lo anterior, el tema de GC toma cada vez mayor relevancia tanto para las empresas a nivel mundial como para las compañías en Chile, debido a la globalización en la que nos encontramos y que ha llevado a un cierto grado de estandarización en diversas áreas de la economía, con el objetivo de poder clasificar, comparar y discernir entre las mejores opciones en el mercado (Silva, 2015) de tal forma de poder mantener los niveles de competencia entre los mercados de capitales y entregar los apropiados controles a inversionistas globales cada vez más involucrados en estos mercados (García, 2014).

A lo anterior se suma, la escaza investigación en torno al tema de GC, tanto en Latinoamérica como en Chile, lo que abre diversas dudas en relación a como se está llevando la adopción de este sistema con sus diferentes herramientas y mecanismos que tienen como objetivo resguardar y satisfacer los derechos y requerimientos de los stakeholders. Otra motivación que hace interesante el desarrollo de este tema, es el rol activo que ha tomado la SVS, en exigir a las empresas chilenas, que divulguen en forma clara y explícita, su adopción de GC, a través de la emisión de la NCG N³41, en el año 2012, la cual fue prontamente derogada, para aumentar el grado de detalle y divulgación a través de la nueva NCG $N^{\circ} 385$, sobre gobiernos corporativos emitida el 08 de junio del 2015, lo que hace que nuestra investigación se realice sobre los primeros resultados de la NCG $\mathrm{N}^{\circ} 385$, otorgando un mayor valor a la información y resultados obtenidos.

En general podemos indicar que nuestra investigación pretende ser un aporte al tema de GC, que ha querido impulsar el estado chileno a través de diferentes normas, incluso leyes. Además, nuestra investigación, incorpora nuevas variables al análisis de GC, como es la búsqueda de la relación entre la adopción de prácticas relacionadas con GC y variables de índole financiera como: el indicador Q de Tobin, la Rentabilidad neta, la Variación de la rentabilidad neta, la Rentabilidad sobre el patrimonio, el Indicador Dow Jones Sustainability Index Chile y el IPSA.

\section{Formulación de objetivos}

El Objetivo principal del estudio es determinar y analizar el grado de adopción y homogeneidad de las prácticas de GC implementadas por las empresas chilenas inscritas en la SVS y si esta adopción se ve afectada por variables de desempeño financiero.

Así como estudios previos han analizado la relación entre la adopción de GC y el sector de pertenencia (García, 2014; Moraga et al, 2015), esperamos que los sectores que han sido relacionados con mayor adopción de prácticas de RSE, también sean los con mayor adopción de 
prácticas de GC. También se considera relevante establecer la relación con algún indicador de tipo financiero, que haya sido utilizado por otros autores e incorporarlo a la realidad del mercado chileno.

De acuerdo a lo anterior para la presente investigación nos hemos planteado los siguientes objetivos específicos los cuales están en línea y tributan al objetivo general:

- Primer objetivo específico: Determinar el grado de adopción de las prácticas de GC en las empresas chilenas, a través de un índice de adopción creado con la información aportada por las empresas cotizadas.

- Segundo objetivo específico: Determinar los sectores y subsectores económicos con mayor y menor grado de adopción de las prácticas de GC.

- Tercer objetivo específico: Determinar la homogeneidad interna en la adopción de las prácticas de GC para cada sector y subsector.

- Cuarto objetivo específico: Analizar la existencia de una relación entre el grado de adopción general de las prácticas de GC y variables relacionadas con el desempeño financiero.

\section{Formulación de Hipótesis}

Son diversos los estudios empíricos que relacionan el sector económico como factor explicativo de una mayor revelación de información sobre RSE, Moneva y Llena (2000), Archel y Lizarraga (2001), Da Silva y Aibar (2011) y Calvo (2016), lo cual nos puede dar un indicio de que suceda algo similar con los estudios que relacionan el grado de adopción de GC con el sector de pertenencia entre los que podemos destacar autores como Rejón (2011) y Moraga et al. (2015).

Los sectores y subsectores económicos, están determinados por la SVS, estableciendo tres sectores económicos y quince subsectores, lo que nos plantea, que para alcanzar los objetivos generales y específicos de esta investigación deberemos establecer las siguientes hipótesis:

$\mathbf{H}_{1}$ : Existe homogeneidad en el grado de adopción de prácticas de GC, de las empresas cotizadas pertenecientes a un mismo sector o subsector económico.

$\mathbf{H}_{2}$ : El sector o subsector económico al que pertenecen las empresas cotizadas incide en el grado de adopción de prácticas de GC.
Desempeño Económico. Las investigaciones previas sobre la adopción de GC han relacionado varios factores económicos, dentro de los que destacan índices de solvencia, rendimiento financiero conocido como ROE (return on equity), en estas, la variable ingresos operacionales, tiene una destacada participación, esta variable los autores la relacionan con el comportamiento del consumidor, debido a que es sensible a la percepción que tiene el consumidor del comportamiento responsable de la empresa (Hawas y Tse, 2015; Krafft et al., 2013). Por otra parte, existen autores que coinciden en medir el desempeño económico, con variables de mercado, entre ellas el resultado de la empresa (García, 2014; Díaz, 2007), el valor accionario, el valor de la empresa (Z-altman) y la Q de Tobin (Burneo y Lizarzaburu, 2016; Rejón, 2011; Wang, 2015), ambas ponen en el centro del análisis al inversionista.

También la pertenencia a un indicador como el Dow Jones Sustainability Index o al Índice de Precios Selectivo de Acciones, ha sido utilizado por diversos autores que han considerados estos dos conceptos como variables para contrastar diversas hipótesis (Correa-García et al., 2016; Lefort y Walker, 2002; Silva, 2015; Alfaro y Silva, 2008; García, 2014; Terán y Molina, 2005), ya que estos indicadores están conformados por entidades que presentan diversas características en común, las cuales pueden ser consideradas como variables explicativas para ciertos resultados. Los anteriores conceptos inspiran la formulación de la siguiente hipótesis:

$\mathbf{H}_{3}$ : El grado de adopción de las prácticas de GC se relaciona con el desempeño financiero de las empresas cotizadas.

\section{METODOLOGÍA, MATERIAL Y MÉTODOS}

La base de datos analizada en este trabajo está compuesta por las sociedades cotizadas en la bolsa de valores de Chile en el año 2015, las cuales están obligadas a inscribirse en la SVS y aquellas empresas que voluntariamente están inscritas en la SVS. Para la selección de las entidades objeto de estudio, se ha tenido en cuenta que dieran respuesta a lo solicitado por la NCG N³85 emitida por la SVS, con las prácticas de GC adoptada al 31 de diciembre del 2015.

El total de empresas que debían responder al cuestionario corresponde a 195 empresas de las cuales 2 no respondieron o no entregaron la in- 
formación, además cuatro empresas que no estaban obligadas a responder, lo hicieron en forma voluntaria, por lo cual la base de datos quedó conformada por 197 instituciones, de las cuales 159 cotizan sus acciones en la Bolsa de Comercio de Santiago habitualmente.

Para obtener las respuestas de las empresas, se ingresó a la página de la SVS, se buscó cada una de las empresas que componen la investigación, accedimos al apartado "Prácticas de Gobiernos Corporativos" y tabulamos las respuestas al cuestionario aportado por estas empresas para el año 2015 en una planilla Excel, considerando las 99 consultas realizadas por la NCG N`385, para luego ser traspasada al programa estadístico SPSS v24, se debe considerar que el formulario contiene respuestas dicotómicas SI o $\mathrm{NO}$, a las cuales se les asignaron uno (1) y cero (0) respectivamente.

Una vez tabulada las respuestas de las instituciones, se procedió a confeccionar un Indicador de Adopción de Gobierno Corporativo por Empresa (IAGCEx), aplicándose la siguiente ecuación.

$$
\begin{gathered}
\text { IAGCEx }=(\text { RAS1i })^{*} 0,52+(\text { RAS2i })^{*} 0,22+ \\
(\text { RAS3i })^{*} 0,22+(\text { RAS4i })^{*} 0,04
\end{gathered}
$$

\section{Donde}

$R A S x i=$ Es el promedio de las respuestas positivas a las preguntas que conforman cada una de las 4 secciones del cuestionario.

En cuanto a las herramientas utilizadas, estas fueron pruebas para determinar la normalidad (Kolmogorov-Smirnov) para posteriormente calcular el coeficiente de variación (CV) que nos servirá para determinar la homogeneidad interna de cada uno de los sectores y subsectores económicos en que está dividida nuestra base de datos.

Para la elección de los ratios utilizados como medida de las variables independientes, sector de actividad, subsector y valor de empresa, se ha procedido previamente a realizar una revisión de la literatura, que facilite la selección de los mismos, y la relación esperada bajo el contexto chileno.

\section{El Modelo de Valoración de la Q de Tobin}

La $Q$ de Tobin (Tobin, 1969) se basa en una teoría del financiamiento de la inversión a través de la Bolsa de Valores que fue publicada por
James Tobin en el Journal of Money Credit and Banking (Casas et al., 2011). Este modelo puede ser definido como el ratio entre el valor de mercado asignado a todos los activos de una firma, considerando los de carácter intangible, como por ejemplo, el potencial de crecimiento, calidad de gestión, poder de mercado, entre otros, versus su costo de reposición. Sin embargo, existen variantes metodológicas para estimar esta ratio (Burneo y Lizarzaburu, 2016)

La Q de Tobin es considerada también como una de las cuatro teorías determinante de la inversión (Carrasco et al., 2005) que plantea que la inversión depende del cuociente entre el valor de mercado del capital y su costo de reposición (ratio Q). Según los autores Montoro y Navarro (2010), la metodología más adecuada y empleable en términos de calcular el potencial de rendimiento que tiene la economía es la de Chung y Pruitt (1994), el modelo que emplearon como base fue modificado tomando en cuenta una forma aún más sencilla, la misma que se expresa como simplemente el cociente del valor de mercado de la empresa, como ya se ha explicado anteriormente este concepto se puede obtener por medio de la capitalización bursátil de la entidad en el mercado y del valor en libros de la totalidad de activos de una empresa. Para Montoro y Navarro (2010) la Q de Tobin aplicable se expresa de la siguiente manera:

$$
\mathrm{Q} \text { simple }=\frac{\mathrm{VM}(\mathrm{AC})}{\mathrm{VL}(\mathrm{TA})}
$$

\section{Margen de rentabilidad neta de las empresas}

Podemos describir la utilidad neta de una empresa, como la utilidad (pérdida) neta presentada en el Estado de Resultado correspondiente a un período en particular, cabe señalar que la utilidad neta no constituye un buen proxy de la capacidad de generación de flujos de efectivo de un negocio, ya que se ve afectada por la deducción de gastos contables tales como: depreciación del activo fijo, amortización del activo intangible, amortización menor valor de inversiones, diferencias de tipo de cambio y amortización mayor valor de inversiones. Además, la utilidad neta se ve afectada por flujos de ingresos y egresos que tienen un carácter transitorio como, por ejemplo, otros ingresos fuera de la explotación, otros egresos fuera de la explotación, diferencias de tipo de cambio e ítems extraordinarios. Finalmente, la utilidad del período se calcula de acuerdo al principio de devengado, lo que constituye una diferencia fundamental con 
el flujo de efectivo (Cornejo y Díaz, 2016).

Para nuestra investigación, la utilidad neta será utilizada en dos aspectos, la primera dice relación con la utilidad neta generada por las empresas cotizadas durante el año 2015, que obtendremos del Estado de Resultado presentado a la SVS, y que será representado como el cociente entre la utilidad neta del ejercicio sobre el ingreso operacional neto de la entidad (Sanz, 2002), la segunda forma de utilización de esta variable, se refiere a determinar la variación de la misma entre los periodos 2015 y 2016, como se explica en las siguientes formulas:

Formula 1 Ratio Margen de Rentabilidad neta.

Margen de Rentabilidad Neta $=\frac{\text { Resultado de explotación }}{\text { Ventas netas }}$

Fuente: Elaboración propia.

Formula 2 Variación de Rentabilidad de venta.

Variación rentabilidad $=$ Resultado de explot. 2016 - Resultado de explot. 2015

Resultado de explotación 2015

Fuente: Elaboración propia.

\section{La Rentabilidad sobre el patrimonio (ROE)}

La rentabilidad financiera o rentabilidad sobre el patrimonio, más conocido como ROE por sus siglas en inglés Return on equity, relaciona el beneficio económico con los recursos necesarios para obtener la ganancia. Para nuestra investigación hemos considerado el ratio de la rentabilidad financiera según lo descrito por (Sanz, 2002) quien describe el ratio como el cociente entre el beneficio después de impuestos y el patrimonio neto:

Formula 3 Ratio de Rentabilidad sobre el patrimonio.

$$
\text { Rentabilidad sobre el patrimonio }=\frac{\text { Beneficio Neto }}{\text { Patrimonio Neto }}
$$

Fuente: Elaboración propia.

\section{Indicador del Dow Jones Sustainability Index Chile}

El índice Dow Jones Sustainability Index Chile (DJSI Chile) fue instaurado desde el año 2015 en Chile, aunque sus orígenes se remontan al año 1999, creado por Standard and Poor's Dow Jones (S\&P) y RobecoSAM, y es considerado el primer índice global de calificación de rendimiento financiero e integración de valoraciones. El índice tiene dos vertientes: el índice global y el índice de mercados emergentes, el primero selecciona empresas que cotizan en la Bolsa de Valores de Nueva York y el segundo mide el rendimiento de las empresas en países en vía de desarrollo que solo cotizan en bolsas de valores locales (Correa-García et al., 2016). En nuestra investigación, intentaremos relacionar, que las $21 \mathrm{em}-$ presas que componen actualmente el DJSI Chile están motivadas a adoptar en mayor grado las prácticas de GC. 


\section{Indicador del Índice de Precios Selectivo de Acciones (IPSA)}

El IPSA es descrito por la SVS en su página $\mathrm{web}^{3}$, como un indicador que considera las variaciones de precios de las 40 compañías más liquidas, o dicho de otra forma, con mayor presencia bursátil que cotizan en la Bolsa de Comercio de Santiago. La selección de las empresas que componen el IPSA se realiza trimestralmente. El índice se construye ponderando el patrimonio bursátil, que se refiere a la multiplicación de la cantidad de acciones emitidas por la empresa versus el valor en bolsa de dicha acción, ajustado por el free-float, que se refiere a las acciones que se encuentran en circulación, disponible para su compra y venta y que no considera las acciones que se encuentran en los grupos controladores.

Desde los inicios del IPSA en el año 1977, han sido diversos autores que han utilizado este indicador, como referente para realizar los más diversos análisis (Lefort y Walker, 2002; Silva, 2015; Alfaro y Silva, 2008; Terán y Molina, 2005), básicamente porque reúne a las empresas más importantes del mercado bursátil chileno, así también ha sido utilizado para relacionar este indicador con la aplicación de RSE (Marin et al., 2012; Morales y Jarne, 2009) y con gobierno corporativo (Silva, 2015; García, 2014).

En nuestra investigación utilizaremos este indicador, para establecer si las 40 empresas que componen este indicador, realizan una mayor adopción que aquellas empresas que estan excluidas del indicador.

\section{RESULTADOS}

En la Tabla 1 podemos observar el porcentaje de empresas que respondió afirmativamente las preguntas referidas a la adopción de las políticas y prácticas de gobierno corporativo, que exigen las cuatro secciones de la NCG N³85. Además del Indicador de Adopción de Gobierno Corporativo por sector y por Subsector.

Tabla 1. Adopción de Gobierno Corporativo por sector y subsector.

\begin{tabular}{|c|c|c|c|c|c|c|c|c|}
\hline & Indicador Adopción GC & & $52 \%$ & $22 \%$ & $22 \%$ & $4 \%$ & $100 \%$ & $100 \%$ \\
\hline Sector & \begin{tabular}{|c|} 
Subsector \\
\end{tabular} & $\begin{array}{c}\text { Sector de } \\
\text { pertenencia }\end{array}$ & Área I & Área II & Área III & Área IV & $\begin{array}{c}\text { Total } \\
\text { Subsector }\end{array}$ & $\begin{array}{c}\text { Total } \\
\text { sector }\end{array}$ \\
\hline agropecuario & Agricultura, ganadería, caza y silv. & 12 & $27,8 \%$ & $24,6 \%$ & $34,1 \%$ & $0,0 \%$ & $27,4 \%$ & \\
\hline agropecuario & Explotación de minas y canteras & 8 & $26,2 \%$ & $31,3 \%$ & $31,3 \%$ & $12,5 \%$ & $27,9 \%$ & \\
\hline agropecuario & Pesca & 10 & $28,6 \%$ & $28,6 \%$ & $34,5 \%$ & $15,0 \%$ & $29,4 \%$ & $28,2 \%$ \\
\hline industrial & Construcción & 9 & $32,9 \%$ & $27,3 \%$ & $42,9 \%$ & $2,8 \%$ & $32,7 \%$ & \\
\hline industrial & Ind. manufactureras metálicas & 10 & $53,1 \%$ & $44,1 \%$ & $52,3 \%$ & $20,0 \%$ & $49,6 \%$ & \\
\hline industrial & Ind. manufactureras no metálicas & 35 & $35,2 \%$ & $30,6 \%$ & $35,6 \%$ & $8,6 \%$ & $33,2 \%$ & \\
\hline industrial & Sum. de Electricidad, Gas y Agua & 25 & $26,7 \%$ & $31,3 \%$ & $43,1 \%$ & $6,0 \%$ & $30,5 \%$ & $34,4 \%$ \\
\hline servicio & Act. Inmobiliarias & 8 & $33,1 \%$ & $25,0 \%$ & $17,6 \%$ & $0,0 \%$ & $26,5 \%$ & \\
\hline servicio & Adm. pública y defensa & 4 & $45,6 \%$ & $43,2 \%$ & $67,0 \%$ & $31,3 \%$ & $49,2 \%$ & \\
\hline servicio & Comercio al por mayor o menor & 13 & $34,7 \%$ & $32,2 \%$ & $50,3 \%$ & $1,9 \%$ & $36,3 \%$ & \\
\hline servicio & Hoteles y restaurantes & 2 & $24,5 \%$ & $29,5 \%$ & $15,9 \%$ & $0,0 \%$ & $22,7 \%$ & \\
\hline servicio & Intermediación financiera & 19 & $23,7 \%$ & $18,7 \%$ & $21,8 \%$ & $2,6 \%$ & $21,3 \%$ & \\
\hline servicio & Otras actividades de servicios & 22 & $31,5 \%$ & $16,9 \%$ & $19,4 \%$ & $5,7 \%$ & $24,5 \%$ & \\
\hline servicio & Servicios sociales y de salud & 9 & $38,3 \%$ & $27,3 \%$ & $46,0 \%$ & $5,6 \%$ & $36,3 \%$ & \\
\hline \multirow[t]{2}{*}{ senvicio } & Transp., almac. y comunicaciones & 11 & $42,2 \%$ & $35,5 \%$ & $52,1 \%$ & $9,1 \%$ & $41,6 \%$ & $30,2 \%$ \\
\hline & Total general & 197 & $32,8 \%$ & $28,4 \%$ & $36,3 \%$ & $7,1 \%$ & $31,6 \%$ & $31,6 \%$ \\
\hline
\end{tabular}

Fuente: Elaboración propia.

La Tabla 1 muestra el grado de adopción general de las 197 empresas consideradas en esta primera parte de la investigación, el cual alcanza a un $31,6 \%$, medido a través de los 15 subsecto- res y los 3 sectores económicos, establecidos por la SVS. Esto demuestra una baja, en relación a los resultados obtenidos por autores como Moraga et al. (2015) en su investigación con la nor-

3 Recuperado de http:/ / www.svs.cl/educa/600/w3-propertyvalue-734.html, el 12.04.2017. 
ma anterior NCG $\mathrm{N}^{\circ} 341$ enfocada en el mismo tema, donde concluyeron un grado de adopción total equivalente al $57 \%$.

\section{Análisis de la homogeneidad por sector económico}

La Tabla 2 muestra un resumen estadístico por sector de pertenencia, considerando el Coeficiente de Variación (CV) como la medición que determina homogeneidad o heterogeneidad de los datos. En este caso, en todos los sectores el $\mathrm{CV}$ es superior a un $30 \%$, por lo que se deduce, que los sectores de pertenencia -agropecuario, industrial y servicio- son heterogéneos internamente. Dicho de otra forma, los tres grupos económicos que componen la investigación presentan una alta dispersión de sus datos, lo que nos permite concluir que el sector económico de pertenencia no es un factor preponderante para establecer un grado de adopción mayor en una institución cotizada en la bolsa.

Tabla 2. Homogeneidad por sector económico.

\begin{tabular}{|l|c|c|c|c|c|c|}
\hline Adopción Sector & $\mathbf{N}$ & $\begin{array}{c}\text { Desviación } \\
\text { estándar }\end{array}$ & Media & Mediana & CV & CV \% \\
\hline Agropecuario & 30 & 21,27383 & 27,9000 & 19,50 & 0,7625028 & $76 \%$ \\
\hline Industrial & 77 & 18,58232 & 34,0130 & 32,00 & 0,5463299 & $55 \%$ \\
\hline Servicio & 90 & 21,52937 & 29,9444 & 25,00 & 0,7189781 & $72 \%$ \\
\hline Total & 197 & 20,41635 & 31,2234 & 28,00 & 0,6538797 & $65 \%$ \\
\hline
\end{tabular}

Fuente: Elaboración propia.

Otra de las preguntas a las cuales esta investigación desea dar respuesta, es al comportamiento $\mathrm{u}$ homogeneidad de los subsectores establecidos por la SVS, para lo cual realizaremos el mismo procedimiento, pero esta vez a los quince subsectores que componen la base de investigación. Los resultados se reflejan en la siguiente Tabla 3.

Tabla 3: Homogeneidad por subsector económico.

\begin{tabular}{|l|r|r|c|c|c|}
\hline \multicolumn{1}{|c|}{ Subsector } & N & $\begin{array}{c}\text { Desviación } \\
\text { Típica }\end{array}$ & Media & CV & CV \% \\
\hline Agricultura, ganadería, caza y silvicultura & 12 & 23,58913 & 27,0833 & 0,8709843 & $87 \%$ \\
\hline Explotación de minas y canteras & 8 & 16,24753 & 27,6250 & 0,5881459 & $59 \%$ \\
\hline Pesca & 10 & 23,88142 & 29,1000 & 0,8206674 & $82 \%$ \\
\hline Construcción & 9 & 18,22087 & 32,3333 & 0,5635326 & $56 \%$ \\
\hline Industrias manufactureras metálicas & 10 & 25,39226 & 49,1000 & 0,517154 & $52 \%$ \\
\hline Industrias manufactureras no metálicas & 36 & 19,08156 & 32,8000 & 0,5817549 & $58 \%$ \\
\hline Suministro de Electricidad, Gas y Agua & 25 & 12,580673 & 30,2400 & 0,4160275 & $42 \%$ \\
\hline Act. inmobiliarias, empresa y de alquiler & 8 & 17,782415 & 26,2500 & 0,6774253 & $68 \%$ \\
\hline Adm. pública y defensa & 4 & 19,465782 & 48,7500 & 0,3992981 & $40 \%$ \\
\hline Comercio al por mayor o menor & 13 & 18,772593 & 35,9231 & 0,5225775 & $52 \%$ \\
\hline Hoteles y restaurantes & 2 & 2,1213203 & 22,5000 & 0,0942809 & $9 \%$ \\
\hline Intermediación financiera & 19 & 18,429731 & 21,1053 & 0,8732291 & $87 \%$ \\
\hline Otras actividades de servicios & 22 & 18,328177 & 24,2727 & 0,7550934 & $76 \%$ \\
\hline Servicios sociales y de salud & 9 & 24,922101 & 35,8889 & 0,6944239 & $69 \%$ \\
\hline Transporte, almac. y comunicaciones & 11 & 28,992476 & 41,1818 & 0,7040115 & $70 \%$ \\
\hline
\end{tabular}

Fuente: Elaboración propia. 
A través de la Tabla 3 podemos establecer que de los 15 subsectores económicos que componen la investigación, solo el subsector "Hoteles y Restaurantes" puede ser considerado homogéneo, por lo cual los otros catorce subsectores, podemos indicar que son heterogéneos, ya que su coeficiente de variación es muy superior al 30\%.

\section{Análisis de correlación entre IAGCT y Q-Tobin}

El Coeficiente de correlación de Spearman es un coeficiente no paramétrico para aquellos grupos de datos que no se ajustan a una distribución conocida, es decir no tienen una distribución normal, lo cual lo hemos obtenido a través de la prueba de normalidad de Kolmogorov-Smirnov realizado en el apartado anterior.

Hemos realizado las pruebas estadísticas no paramétricas que nos permitan establecer si existe relación entre el grado de adopción de las prácticas de gobiernos corporativos por parte de las empresas y la valorización de la empresa medido a través de la $Q$ de Tobin. Debido a que 38 empresas del total que contestaron el cuestionario de la NCG N³85, no cotizaron sus acciones en la bolsa durante el año 2015, la cantidad de datos que pudimos correlacionar disminuye a 159 datos. De esta forma procedemos a realizar el análisis de correlación a través de la prueba no paramétrica con el coeficiente de correlación de Spearman que arroja los resultados expresados en la Tabla 4.

Tabla 4. Correlación de Spearman para IAGCT y Q de Tobin.

\begin{tabular}{|lll|r|r|}
\hline \multicolumn{2}{|c|}{ Correlaciones } & \multicolumn{2}{c|}{ Total Adopc } & Qtobin \\
\hline \multirow{3}{*}{ Rho de Spearman } & \multirow{2}{*}{$\begin{array}{l}\text { Total } \\
\text { Adopc }\end{array}$} & Coeficiente de correlación & 1,000 &, 081 \\
\cline { 3 - 5 } & & Sig. (bilateral) &. &, 312 \\
\cline { 3 - 5 } & $\mathrm{N}$ & 159 & 159 \\
\hline
\end{tabular}

Fuente: Elaboración propia.

El supuesto de la Prueba Spearman, afirma que no existe correlación entre el Indicador Adopción de Gobierno Corporativo Total (IAGCT) y la Q- Tobin. Esto es confirmado, pues la significancia o p-valor supera al valor de significancia limite $(0,05)$, o grado de error que se puede soportar, por lo cual podemos afirmar con un 95\% de confianza, que no existe relación entre ambas variables.

\section{Análisis de correlación entre IAGCEx y Rentabilidad de las ventas}

En cuanto a relacionar la variable de adop- ción de GC versus la rentabilidad neta obtenida por las entidades durante el año 2015 medido a través del ratio financiero Rentabilidad de las ventas (MUN 2015) que hemos obtenidos a través de los estados financieros presentados por las empresas bajo análisis a la SVS para el año comercial 2015.

Como hemos indicado en apartados precedentes los datos bajo análisis, no mantienen normalidad por lo cual aplicamos la prueba no paramétrica llamada Rho de Spearmann, para medir la relación entre ambas variables, ya explicadas. El resumen de los datos procesados se representa en la siguiente Tabla 5.

Tabla 5. Correlación de Spearman entre IAGCEx y Margen de Rentabilidad Neta.

\begin{tabular}{|l|l|l|c|c|}
\hline \multicolumn{5}{|c|}{ Correlaciones } \\
\hline \multirow{2}{*}{ Rho de Spearman } & \multirow{2}{*}{ Total Adopc } & Coeficiente de correlación & Total Adopc & MUN 2015 \\
\cline { 3 - 5 } & & Sig. (bilateral) & 1,000 &,- 108 \\
\cline { 3 - 5 } & & N & 159 &, 216 \\
\hline
\end{tabular}

Fuente: Elaboración propia. 
La Tabla precedente, nos muestra que debido a que el grado de significancia es mayor que 0,05 el cual nos hemos puesto como error tolerable, debemos aceptar la hipótesis nula que indica que existe independencia entre las variables analizadas.

\section{Análisis de correlación entre IACGEx y Variación de la rentabilidad}

Posteriormente hemos procedido a revisar si existe una relación entre la variación de la utilidad neta y el grado de adopción de GC por empresa, medido a través del Indicador de Adopción de Gobierno Corporativo por Empresa (IACGEx) analizado en los capítulos anterio- res, para tal efecto hemos tomado la variación de la rentabilidad neta entre el periodo diciembre 2015 y diciembre 2016, que hemos obtenidos a través de los estados financieros presentados por las empresas bajo análisis a la SVS para el año comercial 2016.

Como hemos indicado en apartados precedentes los datos bajo análisis, no mantienen normalidad por lo cual aplicamos la prueba no paramétrica llamada Rho de Spearmann, para medir la relación entre ambas variables, ya explicadas. El resumen de los datos procesados se representa en la siguiente tabla 6 . La siguiente tabla muestra la relación entre las dos variables descritas:

Tabla 6. Correlación de Spearman entre IAGCEx y Variación de rentabilidad.

\begin{tabular}{|c|c|c|c|c|}
\hline \multicolumn{5}{|c|}{ Correlaciones } \\
\hline & & & Total Adopc & Variación Util \\
\hline \multirow[t]{3}{*}{ Rho de Spearman } & \multirow[t]{3}{*}{ Total Adopc } & Coeficiente de correlación & 1,000 &, 087 \\
\hline & & Sig. (bilateral) & . & ,306 \\
\hline & & $\mathrm{N}$ & 159 & 141 \\
\hline
\end{tabular}

Fuente: Elaboración propia.

La Tabla precedente, nos muestra que debido a que el grado de significancia es mayor que 0,05 el cual nos hemos puesto como error tolerable, debemos aceptar la hipótesis nula que indica que existe independencia entre las variables analizadas.

\section{Análisis de correlación entre IAGCEx y rentabilidad sobre el patrimonio neto (ROE)}

Además, hemos analizado la relación entre el grado de adopción de gobierno corporativo por empresa y la rentabilidad sobre el patrimonio neto (ROE), obtenida para el año 2016, dicho análisis se ha realizado con la ya mencionada prueba estadística Rho de Spearmann, la cual nos arroja los resultados que se exponen en la Tabla 7:

Tabla 7: Correlación de Spearman entre IAGCEx y ROE.

\begin{tabular}{|l|l|l|c|c|}
\hline \multicolumn{5}{|c|}{ Correlaciones } \\
\hline \multirow{2}{|c|}{ Rho de Spearman } & \multirow{2}{*}{ Sumatoria } & Coeficiente de correlación & Sumatoria & \multicolumn{1}{c|}{ Roe } \\
\cline { 3 - 5 } & & Sig. (bilateral) & $\cdot$ &,- 012 \\
\cline { 3 - 5 } & & N & 197 &, 867 \\
\cline { 3 - 5 } & &
\end{tabular}

Fuente: Elaboración propia. 
De acuerdo a los resultados del coeficiente de Spearman, el grado de significancia es mayor que el 0,05 que nos hemos puesto como error tolerable, por lo cual se rechaza la hipótesis alternativa y se acepta la hipótesis nula que indica que existe independencia entre las variables analizadas, por lo cual podemos concluir que no existe una relación entre las dos variables que hemos analizado, el hecho de tener un mayor grado de adopción de gobierno corporativo no es causal de tener mejor rendimiento financiero.

\section{Análisis de correlación entre IAGCEx y la pertenencia al Índice Dow Jones Sustainability Index Chile}

El índice Dow Jones Sustainability Chile
Index (DJSI Chile) reúne a las empresas que se consideran líderes en sostenibilidad, para tal clasificación la empresa RobecoSAM (empresa especialista en la investigación sobre sustentabilidad) realiza una encuesta anual denominada Corporate Sustainability Assessment (CSA) que considera tres aspectos el ambiental, el social y de buen gobierno corporativo, de esta forma establece que aquellas empresas con mayor puntaje, sean partícipes de este indicador.

En el siguiente apartado hemos querido analizar si existe una relación entre el IAGCEx y las empresas que pertenecen al DJSI Chile. Para medir el grado de relación hemos vuelto a aplicar Rho de Spearman, los resultados se exponen en la Tabla 8 .

Tabla 8: Correlación de Spearman entre IAGCEx y pertenencia al DJSI.

\begin{tabular}{|c|c|c|c|c|}
\hline \multicolumn{5}{|c|}{ Correlaciones } \\
\hline & & & Total Adopc & DJS \\
\hline \multirow[t]{3}{*}{ Rho de Spearman } & \multirow[t]{3}{*}{ Total Adopc } & Coeficiente de correlación & 1,000 & ,192* \\
\hline & & Sig. (bilateral) & . & ,016 \\
\hline & & $\mathrm{N}$ & 159 & 159 \\
\hline
\end{tabular}

Fuente: Elaboración propia.

De acuerdo a los resultados del coeficiente de Spearman, el grado de significancia es menor que el 0,05 que nos hemos puesto como error tolerable, por lo cual tenemos evidencia necesaria para aceptar la hipótesis alternativa que nos señala que existen una relación entre el hecho de pertenecer al DJSI Chile y el grado de adopción de gobierno corporativo total empresa.

\section{Análisis de correlación entre IAGCEx y la pertenencia al Índice Precio Selectivo de Acciones (IPSA)}

Entre los análisis realizados, hemos procedido a identificar a aquellas empresas que son parte del IPSA y las que no pertenecen a dicho índice, para posteriormente relacionar estos dos grupos con su grado de adopción de GC y establecer si existe una diferencia significativa entre aquellas empresas que pertenecen a dicho índice y su grado de adopción de GC medido a través del IAGCEx. Los resultados se exponen en la Tabla 9 . 
Tabla 9: Correlación de Spearman entre IAGCEx y pertenencia al IPSA.

\begin{tabular}{|c|c|c|c|c|}
\hline \multicolumn{5}{|c|}{ Correlaciones } \\
\hline & & & Sumatoria & IPSA \\
\hline \multirow[t]{3}{*}{ Rho de Spearman } & \multirow[t]{3}{*}{ Sumatoria } & Coeficiente de correlación & 1,000 & $278^{* *}$ \\
\hline & & Sig. (bilateral) & . & ,000 \\
\hline & & $\mathrm{N}$ & 197 & 197 \\
\hline
\end{tabular}

Fuente: Elaboración propia.

De acuerdo a los resultados del coeficiente de Spearman, podemos decir que aceptamos la hipótesis alternativa que indica que existen una relación entre las dos variables que hemos analizado, dicha relación la podemos clasificar como baja relación ya que llega a un 0,278.

\section{DISCUSION, CONCLUSIONES}

Al tabular las respuestas al cuestionario exigido por la NCG $\mathrm{N}^{\circ} 385$, y entregadas por las instituciones cotizadas en bolsa, podemos observar que existe un compromiso por parte de dichas empresas en responder el cuestionario en forma objetiva, considerando que a diferencia de otros autores que han debido obtener la información a través de diversos canales, y diversas fuentes de información como: páginas web, reportes de sustentabilidad, y estados financieros entre otros, nuestra investigación se basa en la información aportada por la fuente primaria que se refiere a la propia empresa.

De los resultados obtenidos podemos concluir que las empresas cotizadas en la bolsa de valores chilena, mantiene un bajo grado de adopción de prácticas relacionadas con buen GC que hemos establecido en $31,6 \%$ para el año 2015 y que este ha disminuido en comparación al grado de adopción medido a través de la NCG $\mathrm{N}^{\circ}$ 341 realizado en el año 2014 con los datos del año 2013 por autores como Moraga et al (2015) y García (2014), esto también deja entrever que la NCG N 385 del año 2015 es más exigente y exhaustiva que la NCG N 341 del año 2012.

El segundo objetivo específico se refiere a determinar los sectores y subsectores económicos con mayor y menor grado de adopción, la Tabla 1 , donde se puede establecer que el sector económico con mayor adopción corresponde al sector industrial con $34,4 \%$, lo cual es bastante bajo con- siderando que en la NCG N 341 llegó a un 57\% (Moraga et al., 2015)

Posteriormente, de la misma Tabla 1 podemos establecer claramente a través de la columna del grado de adopción de prácticas de GC por subsector, cuales son los subsectores con mayor adopción, entre los que destacan el subsector de "industrias manufactureras metálicas" $(49,6 \%)$, el subsector "administración pública" $(49,2 \%)$ y en tercer lugar el subsector "Transporte, almac. y comunicaciones" $(41,6 \%)$. Por el contrario, en los subsectores con menor adopción, podemos destacar tres subsectores, el subsector "Hoteles y restaurantes" (22,7\%), "Intermediación financiera" $(21,3 \%)$ y "Otras actividades de servicios" $(24,5 \%)$.

\section{Conclusiones sobre las hipótesis relativas al sector de actividad}

Con los resultados obtenidos, podemos dar respuesta a la hipótesis $H_{1}$ que establece la homogeneidad de adopción de las prácticas de GC por sector y subsector económico. En este caso, a la luz de los antecedentes y análisis realizados utilizando entre ellos el coeficiente de variación, podemos concluir que existe un alto grado de heterogeneidad en cada uno de los sectores económicos, promediando un $65 \%$ según lo que muestra la Tabla 2, mucho menor al obtenido en el año 2013 en el cual se establecía una alta homogeneidad que alcanzaba el 18,95\% (Moraga et al., 2015)

Posteriormente, se realiza un análisis a los quince subsectores, a los cuales se aplicó los mismos cálculos estadísticos para obtener el CV de cada uno de los subsectores como queda reflejado en la Tabla 3, con este análisis podemos concluir que los subsectores son casi en su totalidad heterogéneos internamente.

Dentro del cuarto objetivo específico, la hipótesis $H_{2}$ apunta a establecer si el sector y sub- 
sector de pertenencia, inciden en el grado de adopción de prácticas de GC, dicha hipótesis es rechazada, ya que debido a la alta heterogeneidad demostrada para cada sector y subsector, podemos establecer que el grado de adopción de estas prácticas no está condicionada por esta variable.

\section{Conclusiones sobre las hipótesis relativas a las variables financieras}

En este caso la conclusión general relativa a los indicadores financieros la debemos separar en dos grandes grupos, las que dicen relación con los ratios financieros $(\mathrm{Q}$ de Tobin, Margen de Rentabilidad neta, variación de la rentabilidad y ROE) y por otra parte una conclusión referida a la pertenencia a indicadores selectivos en cuanto a su confección (DJSI Chile e IPSA). En el primer grupo hemos contrastado a través de herramientas estadísticas que no existe relación, lo cual se traduce que las variables financiera no se ven influenciadas por el grado de adopción de prácticas de GC, esto puede deberse a que los inversionistas desconocen aún la posibilidad de acceder a la información de las practicas adoptadas por la entidad referidas a GC, también podemos establecer que la NCG N³85 es nueva en cuanto a su emisión (2015) y que aún es desconocida por los diversos stakeholders pero podemos esperar que en los siguientes años, se genere un mayor conocimiento de la norma y mayor difusión por parte de las entidades comprometidas con mejorar los estándares de GC en Chile. Lo anterior, otorga un valor agregado a nuestra investigación, ya que a través de esta hemos podido ayudar a la difusión de la norma y sus resultados, y proporcionar una fuente de información más confiable y validada a través de una metodología científica.

Finalmente, en relación a las pruebas de correlación con la pertenencia a indicadores selectivos en cuanto a su confección hemos contrastado a través de herramientas estadísticas que existe relación, lo que implica que un inversionista $\mathrm{u}$ otro grupo de interés relacionado con la entidad, puede utilizar estos grupos de indicadores, ya sea, el DJSI Chile como el IPSA, como una variable válida para preferir a aquellas empresas que resguardan de mejor manera sus intereses, a través de la adopción de mayores prácticas de GC, esta conclusión es importante desde el punto de vista de las investigación, ya que hemos podemos dar continuidad y aportar nuevos resultados a investigaciones anteriores sobre el mismo tema (Silva, 2015; García, 2014; Moraga et al., 2015).

\section{REFERENCIAS BIBLIOGRÁFICAS}

Alfaro, R., \& Silva, C. (Noviembre de 2008). Volatilidad de Indices Accionarios: El caso del IPSA. Cuadernos de Economía, 45, 217-233.

Archel, P., \& Lizarraga, F. (2001). Algunos determinantes de la información medioambiental divulgadas por las empresas españolas cotizadas. Revista de Contabilidad, 4(7), 129-153.

Burneo, K., \& Lizarzaburu, E. (2016). Gobierno Corporativo en Mercados Gobierno Corporativo en Mercados de Lima - BVL. Globalización, Competitividad y Gobernabilidad de Georgetown/Universia, 57-115.

Calvo, E. (2016). Sostenibilidad ambiental y responsabilidad social de las sociedades cotizadas en el mercado bursátil español. Universidad Europea, Facultad de Ciencias Sociales y de la Comunicación, Madrid.

Carrasco, O. F., Johnson, C. A., \& Núñez, H. A. (2005). Determinantes de la inversión a nivel de la empresa: un análisis de panel para Chile. Estudios de Administración, 12(1), p87-122.

Casas Hernández, D. R., \& Combita Mora, G. (2011). Diagramas de fase. Lasalle.

Chung, K. H., \& Pruitt, S. W. (1994). A Simple Approximation of Tobin's q. Financial Management Association International, 70-74.

Cornejo, E., \& Díaz, D. (20 de Junio de 2016). Medidas de Ganancia: EBITDA, EBIT, Utilidad Neta y Flujo de Efectivo. Obtenido de http: / / repositorio. uchile.cl/: http://www.repositorio.uchile.cl/ handle/2250/127394

Correa-García, J., Vásquez-Arango, L., \& SotoRestrepo, Y. (2016). Reportes integrados y generación de valor en empresas colombianas incluidas en el Índice de Sostenibilidad Dow Jones. Cuaderno de Contabilidad, 73-108.

Da Silva, S., \& Aibar, B. (2011). Factores determinantes del grado de información medioambiental divulgada en las grandes empresas que operan en Portugal: Un analisis univariante. Re- 
vista de Administração e Contabilidade da Unisinos, 8(1), 3-19.

Díaz, P. (2007). Un índice de transparencia financiero-contable para las empresas chilenas. $C A$ PIC REVIEW, 5(1).

García, R. (2014). Norma de Información sobre Prácticas de Gobierno Corporativo en Chile. Superintendencia de Valores y Seguros, 1-30.

Hawas, A., \& Tse, C.-b. (2015). How Corporate Governance Affects Investment Decisions of Major Shareholders in UK Listed Companies: Has the Recent Credit Crunch Changed the Game? Journal of Accounting, Auditing \& Finance, 31(1), 100-133.

Krafft, J., Qu, Y., Quatraro, F., \& Ravix, J.-L. (2013). Corporate governance, value and performance of firms: new empirical results on convergence from a large international database. Industrial and Corporate Change, 23(2), 361-397.

Lefort, F., \& Walker, E. (Abril de 2002). Cambios Estructurales e Integración. Discusion y Analisis del Mercado Accionario Chileno. Cuadernos de economía, 39(116).

Marin, M., Maldonado, S., \& Sanmiguel, L. (2012). Análisis empírico de la divulgación de información social en Chile: un estudio en empresas cotizadas. Economía, Gestión y Desarrollo(13), 121-153.

Moneva, J., \& Llena, F. (2000). Enviromental disclosures in the annual reports of large companies in Spain. European Accounting Review, 9(1), 7-29.

Montoro, C., \& Navarro, A. (2010). Estimación de la $Q$ de Tobin para la economía peruana. Revista Estudios Económicos, 19, 33-45.

Morales, F., \& Jarne, J. (2009). E-gobierno corporativo: evidencia en Chile sobre la divulgación de información en internet. Capic Review, 45-62.

Moraga, H., Rossi, M. \& Navarrete, J. (2015) Adopción de Políticas y Prácticas de Gobiernos Corporativos de las empresas Chilenas. Revista Ruta, 17, 25-38.

Rejón, M. (2011). Integrated Reporting: Una mejora sustancial en la información empresarial. Gestión, 11-14.
Rivero, P. (2005). Responsabilidad social y gobierno corporativo: información y transparencia. Revista Asturiana De Economía-Rae, 34, 9-29.

Sanz, C. (2002). Guía de los principales ratios. Acciones e Investigaciones Sociales(14).

Silva, B. (2015). Gobiernos Corporativos en Chile: Estudio sobre el cumplimiento de los principios de la OCDE por las empresas chilenas en 2001-2008 y 2009-2013. Universidad Autónoma de Madrid. Madrid: Universidad Autónoma de Madrid.

Terán, K., \& Molina, O. (2005). Simulación eficiente del valor en riesgo de un portafolio de acciones del IPSA: un análisis de componentes principales. Revista Investigación \& Desarrollo, 1(5).

Tobin, J. (1969). A General Equilibrium Approach To Monetary Theory. Journal of Money, Credit and Banking, 1(1), 15-29. Obtenido de http:/ / www. jstor.org/stable/1991374

Wang, M.-C. (2015). Value Relevance of Tobin's $\mathrm{Q}$ and Corporate Governance for the Taiwanese Tourism Industry. Journal of Business Ethics, 130(1), 223-230. 